\title{
Characterization and Enumeration of Good Punctured Polynomials over Finite Fields
}

\author{
Somphong Jitman, ${ }^{1}$ Aunyarut Bunyawat, ${ }^{2}$ Supanut Meesawat, ${ }^{2}$ \\ Arithat Thanakulitthirat, ${ }^{2}$ and Napat Thumwanit ${ }^{2}$ \\ ${ }^{1}$ Department of Mathematics, Faculty of Science, Silpakorn University, Nakhon Pathom 73000, Thailand \\ ${ }^{2}$ Department of Mathematics, Mahidol Wittayanusorn School, Nakhon Pathom 73170, Thailand
}

Correspondence should be addressed to Somphong Jitman; sjitman@gmail.com

Received 25 November 2015; Revised 3 March 2016; Accepted 6 March 2016

Academic Editor: Shyam L. Kalla

Copyright ( 2016 Somphong Jitman et al. This is an open access article distributed under the Creative Commons Attribution License, which permits unrestricted use, distribution, and reproduction in any medium, provided the original work is properly cited.

\begin{abstract}
A family of good punctured polynomials is introduced. The complete characterization and enumeration of such polynomials are given over the binary field $\mathbb{F}_{2}$. Over a nonbinary finite field $\mathbb{F}_{q}$, the set of good punctured polynomials of degree less than or equal to 2 are completely determined. For $n \geq 3$, constructive lower bounds of the number of good punctured polynomials of degree $n$ over $\mathbb{F}_{q}$ are given.
\end{abstract}

\section{Introduction}

From the fundamental theorem of algebra, every polynomial over the rational numbers $\mathbb{Q}$ (or over the real numbers $\mathbb{R}$ ) has a root in $\mathbb{C}$. However, it is not guaranteed that a polynomial has a root in $\mathbb{Q}$ or in $\mathbb{R}$. Therefore, for a given polynomial over $\mathbb{Q}($ resp., $\mathbb{R})$, it is of natural interest to determine whether it has a root in $\mathbb{Q}($ resp., $\mathbb{R})$. In general, determining whether a given polynomial has a root in a nonalgebraically closed field is an interesting problem and has been extensively studied (see, e.g., $[1-4]$ ).

In this paper, we introduce punctured forms of a polynomial $f(x)$ over a field $\mathbb{F}$ (see the definition below) and focus on determining whether the punctured parts of $f(x)$ have a root in $\mathbb{F}$. Due to the rich algebraic structures and various applications of polynomials over finite fields (see [5-9] and references therein), their properties such as factorization, root finding, and irreducibility have extensively been studied (see [10-13]). In this paper, we mainly focus on punctured polynomials over a finite field which is not an algebraically closed field. The readers may refer to [5] for more details on finite fields and polynomials over finite fields.
Let $q$ be a prime power and let $\mathbb{F}_{q}$ denote the finite field of $q$ elements. Denote by $\mathbb{F}_{q}^{*}$ the set of nonzero elements in $\mathbb{F}_{q}$. Let

$$
\begin{aligned}
& \mathbb{F}_{q}[x] \\
& =\left\{\sum_{i=0}^{m} a_{i} x^{i} \mid a_{i} \in \mathbb{F}_{q} \forall i=0,1,2, \ldots, m, m \in \mathbb{N} \cup\{0\}\right\}
\end{aligned}
$$

be the set of all polynomials with indeterminate $x$ over $\mathbb{F}_{q}$. Let

$$
\begin{aligned}
\mathbb{F}_{q}[x]_{n} & =\left\{f(x) \in \mathbb{F}_{q}[x] \mid \operatorname{deg}(f(x)) \leq n\right\}, \\
\widehat{\mathbb{F}}_{q}[x]_{n} & =\left\{f(x) \in \mathbb{F}_{q}[x] \mid \operatorname{deg}(f(x))=n\right\}
\end{aligned}
$$

be the set of all polynomials of degree less than or equal to $n$ over $\mathbb{F}_{q}$ and the set of all polynomials of degree $n$ over $\mathbb{F}_{q}$, respectively. 
Given a polynomial $f(x)=\sum_{i=0}^{m} a_{i} x^{i} \in \mathbb{F}_{q}[x]$ of degree $m$, for each $j \in\{0,1,2, \ldots, m\}$, the $j$ th punctured polynomial of $f(x)$ is defined to be

$$
f_{(j)}(x)=\sum_{i=0}^{j-1} a_{i} x^{i}+\sum_{i=j+1}^{m} a_{i} x^{i-1} .
$$

For convenience, by abuse of notation, the degree of zero polynomial is defined to be 0 . Hence, we can write $f_{(0)}(x)=0$ for all constant polynomials $f(x)=a \in \mathbb{F}_{q}$.

A polynomial $f(x) \in \mathbb{F}_{q}[x]$ of degree $m$ is said to be good punctured if $f_{(j)}(x)$ has a root in $\mathbb{F}_{q}$ for all $j \in\{0,1,2, \ldots, m\}$. Otherwise, $f(x)$ is said to be bad punctured. The constant polynomials $f(x)=a \in \mathbb{F}_{q}$ are always good punctured and referred to as trivial good punctured polynomials. A good punctured polynomial is called nontrivial if it is not a trivial good punctured polynomial.

Example 1. Let $f(x)=x^{2}+2 x+2$ be a polynomial in $\mathbb{F}_{3}[x]$. Then $f_{(2)}(x)=2 x+2, f_{(1)}(x)=x+2$, and $f_{(0)}(x)=x+2$. It is not difficult to see that $f(x)$ is good punctured.

Given a positive integer $n$ and a prime power $q$, let $T_{(q, n)}$ and $\widehat{T}_{(q, n)}$ denote the set of good punctured polynomials of degree less than or equal to $n$ over $\mathbb{F}_{q}$ and the set of all good punctured polynomials of degree $n$ over $\mathbb{F}_{q}$, respectively. Precisely,

$$
\begin{aligned}
& T_{(q, n)}=\left\{f(x) \in \mathbb{F}_{q}[x]_{n} \mid f_{(j)}(x) \text { has a root in } \mathbb{F}_{q} \forall 0\right. \\
& \quad \leq j \leq \operatorname{deg}(f(x))\} \\
& \widehat{T}_{(q, n)}=\{f(x) \\
& \left.\quad \in \widehat{\mathbb{F}}_{q}[x]_{n} \mid f_{(j)}(x) \text { has a root in } \mathbb{F}_{q} \forall 0 \leq j \leq n\right\} .
\end{aligned}
$$

By convention, since $\operatorname{deg}(0)=0$, we have $T_{(q, 0)}=\widehat{T}_{(q, 0)}=\mathbb{F}_{q}$ and $\left|T_{(q, 0)}\right|=\left|\widehat{T}_{(q, 0)}\right|=q$.

Remark 2. From the definitions of $T_{(q, n)}$ and $\widehat{T}_{(q, n)}$, we have the following facts:

(1) $\widehat{T}_{(q, n)}$ is a subset of $T_{(q, n)}$.

(2) $T_{(q, n)}=T_{(q, n-1)} \cup \widehat{T}_{(q, n)}$ is a disjoint union for all $n \geq 2$.

(3) $\left|T_{(q, n)}\right|=\left|T_{(q, n-1)}\right|+\left|\widehat{T}_{(q, n)}\right|$ for all $n \geq 2$.

Example 3. Over the finite fields $\mathbb{F}_{2}$, we have

$$
\begin{aligned}
& T_{(2,2)}=\left\{0,1, x^{2}, x^{2}+x, x^{2}+x+1\right\}, \\
& \widehat{T}_{(2,2)}=\left\{x^{2}, x^{2}+x, x^{2}+x+1\right\} .
\end{aligned}
$$

Hence, $\left|T_{(2,2)}\right|=5$ and $\left|\widehat{T}_{(2,2)}\right|=3$, respectively.

In this paper, we focus on the characterization and enumeration of the good punctured polynomials of degree $n$ over $\mathbb{F}_{q}$. The complete characterization and enumeration of good punctured polynomials over the binary field $\mathbb{F}_{2}$ are given in Section 2. In Section 3, good punctured polynomials of degree $n$ over $\mathbb{F}_{q}$, where $q>2$, are studied. The good punctured polynomials of degree less than or equal to 2 over fields $\mathbb{F}_{q}$ are completely determined. Lower bounds of the size of the set of good punctured polynomials of degree greater than 2 are provided as well. Conclusion and some discussions about future researches on punctured polynomials are provided in Section 4.

\section{Good Punctured Polynomials over the Binary Field}

In this section, we focus on good punctured polynomials over the finite field $\mathbb{F}_{2}$. The characterization and enumeration of such polynomials are completely determined.

First, we determine the set $T_{(2, n)}$ of good punctured polynomials of degree less than or equal to $n$ over the binary field $\mathbb{F}_{2}$. It is not difficult to see that $T_{(2,1)}=\mathbb{F}_{2}$. For $n \geq 2$, the set $T_{(2, n)}$ is given as follows.

Theorem 4. Let $n \geq 2$ be a positive integer. Then

$$
T_{(2, n)}=A \cup B \cup C,
$$

where $A=\left\{\sum_{i=0}^{2 k} x^{i} \mid 0 \leq k \leq n / 2\right\}, B=\{x f(x) \mid f(x) \epsilon$ $\mathbb{F}_{2}[x]_{n-1}$ and $\left.f(1)=0\right\}$, and $C=\left\{x^{2} f(x) \mid f(x) \in \mathbb{F}_{2}[x]_{n-2}\right\}$.

Proof. First, we prove that $A \cup B \cup C \subseteq T_{(2, n)}$. Let $g(x) \in$ $A \cup B \cup C$. We distinguish the proof into three cases.

Case $1(g(x) \in A)$. Then $g(x)=\sum_{i=0}^{2 k} x^{i}$ for some $0 \leq k \leq n / 2$. It follows that 1 is a root of $g_{(j)}(x)=\sum_{i=0}^{2 k-1} x^{i}$ for all $0 \leq j \leq$ $2 k$. Hence, $g(x) \in T_{(2, n)}$.

Case $2(g(x) \in B)$. Then $g(x)=x f(x)$ for some $f(x) \epsilon$ $\mathbb{F}_{2}[x]_{n-1}$. We have $g_{(0)}(1)=f(1)=0$ and $g_{(i)}(0)=g(0)=0$ for all $0<i \leq \operatorname{deg}(g(x))$. Hence, $g_{(i)}(x)$ has a root in $\mathbb{F}_{2}$ for all $0 \leq i \leq \operatorname{deg}(g(x))$. Therefore, $g(x) \in T_{(2, n)}$.

Case $3(g(x) \in C)$. Then $g(x)=x^{2} h(x)$ for some $h(x) \epsilon$ $\mathbb{F}_{2}[x]_{n-2}$. It follows that 0 is a root of $g_{(0)}(x)=g_{(1)}(x)=x h(x)$ and $g_{(i)}(x)=x^{2} h_{(i-2)}(x)$ for all $2 \leq i \leq \operatorname{deg}(g(x))$. Therefore, $f_{(i)}(x)$ has a root in $\mathbb{F}_{2}$ for all $0 \leq i \leq \operatorname{deg}(g(x))$. As desired, $g(x) \in T_{(2, n)}$.

On the other hand, let $g(x) \in T_{(2, n)}$. Write $g(x)=$ $\sum_{i=0}^{\operatorname{deg}(g(x))} g_{i} x^{i}$ and consider the following two cases.

Case $1\left(g_{0}=0\right)$

Case $1.1\left(g_{1}=0\right)$. Then $g(x) \in C$.

Case $1.2\left(g_{1}=1\right)$. Then $\operatorname{deg}(g(x)) \geq 1$. Since $g_{(0)}(x)=$ $\sum_{i=0}^{\operatorname{deg}(g(x))-1} g_{i+1} x^{i}$, we have $g_{(0)}(0)=g_{1}=1$. It follows that $0=g_{(0)}(1)=\sum_{i=0}^{\operatorname{deg}(g(x))-1} g_{i+1}=g(1)$. Hence, $g(x) \in B$. 
Case $2\left(g_{0}=1\right)$. Since $g_{(0)}(0)=g_{0}=1$, we have $0=g_{(0)}(1)=$ $\sum_{i=1}^{\operatorname{deg}(g(x))} g_{i}$. Suppose that there exists $1 \leq j<\operatorname{deg}(g(x))$ such that $g_{j}=0$. Since $g_{(j)}(0)=g_{0}=1$ and $g_{(\operatorname{deg}(g(x)))}(0)=g_{0}=1$, we have

$$
\begin{aligned}
& 0=g_{(j)}(1)=\sum_{i=0}^{j-1} g_{i}+\sum_{i=j+1}^{\operatorname{deg}(g(x))} g_{i}, \\
& 0=g_{(\operatorname{deg}(g(x)))}(1)=\sum_{i=0}^{\operatorname{deg}(g(x))-1} g_{i} .
\end{aligned}
$$

It follows that $0=g_{j}=g_{\operatorname{deg}(g(x))}=1$, a contradiction. Hence, $g_{i}=1$ for all $0 \leq i \leq \operatorname{deg}(g(x))$. Since $0=g_{(0)}(1)=$ $\sum_{i=1}^{\operatorname{deg}(g(x))} g_{i}$, the degree of $g(x)$ must be even. We conclude that $g(x) \in A$.

From the two cases, we have $g(x) \in A \cup B \cup C$, and, hence, $T_{(2, n)} \subseteq A \cup B \cup C$.

Therefore, $T_{(2, n)}=A \cup B \cup C$ as desired.

Corollary 5. If $n$ is a positive integer, then

$$
\left|T_{(2, n)}\right|= \begin{cases}2 & \text { if } n=1, \\ 3 \cdot 2^{n-2}+\left\lfloor\frac{n}{2}\right\rfloor+1 & \text { if } n \geq 2 .\end{cases}
$$

Proof. By direct calculation, we have $T_{(2,1)}=\{0,1\}$ and $\left|T_{(2,1)}\right|=2$.

Next, assume that $n \geq 2$. By Theorem 4 , we have

$$
\left|T_{(2, n)}\right|=|A \cup B \cup C|,
$$

where $A=\left\{\sum_{i=0}^{2 k} x^{i} \mid 0 \leq k \leq n / 2\right\}, B=\{x f(x) \mid f(x) \epsilon$ $\mathbb{F}_{2}[x]_{n-1}$ and $\left.f(1)=0\right\}$, and $C=\left\{x^{2} f(x) \mid f(x) \in \mathbb{F}_{2}[x]_{n-2}\right\}$.

Since $A$ and $B \cup C$ are disjoint, by the inclusion-exclusion principle, we have

$$
\left|T_{(2, n)}\right|=|A \cup B \cup C|=|A|+|B|+|C|-|B \cap C| .
$$

Clearly, $|A|=\lfloor n / 2\rfloor+1$ and $|C|=2^{n-1}$. Observe that $x f(x)=$ $x \sum_{i=0}^{n-1} f_{i} x^{i} \in B$ if and only if $\left|\left\{0 \leq i \leq n-1 \mid f_{i}=1\right\}\right|$ is even. Hence,

$$
|B|=2^{n-1} .
$$

It is not difficult to see that

$$
B \cap C=\left\{x^{2} f(x) \mid f(x) \in \mathbb{F}_{2}[x]_{n-2}, f(1)=0\right\},
$$

and, hence,

$$
|B \cap C|=2^{n-2}
$$

Therefore,

$$
\begin{aligned}
T_{(2, n)} & =\left\lfloor\frac{n}{2}\right\rfloor+1+2^{n-1}+2^{n-1}-2^{n-2} \\
& =3 \cdot 2^{n-2}+\left\lfloor\frac{n}{2}\right\rfloor+1
\end{aligned}
$$

as desired.
Next, we determine the set $\widehat{T}_{(2, n)}$ of good punctured polynomials of degree $n$ over the binary field $\mathbb{F}_{2}$. Since $T_{(2,1)}=\mathbb{F}_{2}$, we have $\widehat{T}_{(2,1)}=\emptyset$. For $n \geq 2$, the set $\widehat{T}_{(2, n)}$ can be determined as follows.

Theorem 6. If $n \geq 2$ is a positive integer, then

$$
\widehat{T}_{(2, n)}= \begin{cases}\widehat{A} \cup \widehat{B} \cup \widehat{C} & \text { if } n \text { is even, } \\ \widehat{B} \cup \widehat{C} & \text { if } n \text { is odd, }\end{cases}
$$

where $\widehat{A}=\left\{\sum_{i=0}^{n} x^{i}\right\}, \widehat{B}=\left\{x\left(f(x)+x^{n-1}\right) \mid f(x) \in \mathbb{F}_{2}[x]_{n-2}\right.$ and $f(1)=1\}$, and $\widehat{C}=\left\{x^{2}\left(f(x)+x^{n-2}\right) \mid f(x) \in \mathbb{F}_{2}[x]_{n-3}\right\}$.

Proof. We prove the statement by determining the elements in $T_{(2, n)}$ of degree $n$. Let $A=\left\{\sum_{i=0}^{2 k} x^{i} \mid 0 \leq k \leq n / 2\right\}, B=$ $\left\{x f(x) \mid f(x) \in \mathbb{F}_{2}[x]_{n-1}\right.$ and $\left.f(1)=0\right\}$, and $C=\left\{x^{2} f(x) \mid\right.$ $\left.f(x) \in \mathbb{F}_{2}[x]_{n-2}\right\}$ be defined as in Theorem 4 .

It is not difficult to see that the set of elements in $B$ (resp., $C$ ) of degree $n$ is $\widehat{B}$ (resp., $\widehat{C}$ ).

If $n$ is even, then the set of elements in $A$ of degree $n$ is $\widehat{A}$. In the case where $n$ is odd, the set of elements in $A$ of degree $n$ is empty.

By Theorem 4, the result, therefore, follows.

Corollary 7. If $n$ is a positive integer, then

$$
\left|\widehat{T}_{(2, n)}\right|= \begin{cases}0 & \text { if } n=1, \\ 3 & \text { if } n=2 \\ 3 \cdot 2^{n-3} & \text { if } n \geq 3 \text { is odd } \\ 3 \cdot 2^{n-3}+1 & \text { if } n \geq 4 \text { is even. }\end{cases}
$$

Proof. By direct calculation, we have $\widehat{T}_{(2,1)}=\emptyset$ and

$$
\widehat{T}_{(2,2)}=\left\{x^{2}, x^{2}+x, x^{2}+x+1\right\} .
$$

Hence, we have $\left|\widehat{T}_{(2,1)}\right|=0$ and $\widehat{T}_{(2,2)}=3$.

Next, assume that $n \geq 3$. By Theorem 6 , we have

$$
\left|\widehat{T}_{(2, n)}\right|= \begin{cases}|\widehat{A} \cup \widehat{B} \cup \widehat{C}| & \text { if } n \text { is even, } \\ |\widehat{B} \cup \widehat{C}| & \text { if } n \text { is odd, }\end{cases}
$$

where $\widehat{A}, \widehat{B}$, and $\widehat{C}$ are defined as in Theorem 6. Since $\widehat{A}$ and $\widehat{B} \cup \widehat{C}$ are disjoint, by the inclusion-exclusion principle, we have

$$
\left|\widehat{T}_{(2, n)}\right|=|\widehat{A} \cup \widehat{B} \cup \widehat{C}|=|\widehat{A}|+|\widehat{B}|+|\widehat{C}|-|\widehat{B} \cap \widehat{C}| .
$$

We note that $|\widehat{A}|=1$ and $|\widehat{C}|=2^{n-2}$. if

Since $x\left(f(x)+x^{n-1}\right)=x\left(\sum_{i=0}^{n-2} f_{i} x^{i}+x^{n-1}\right) \in \widehat{B}$ if and only

$$
\left|\left\{i \mid 0 \leq i \leq n-2, f_{i}=1\right\}\right|
$$

is odd, we have

$$
|\widehat{B}|=2^{n-2} .
$$


TABLE 1: Punctured polynomials over $\mathbb{F}_{2}$.

\begin{tabular}{lccccccccccccc}
\hline$n$ & 1 & 2 & 3 & 4 & 5 & 6 & 7 & 8 & 9 & 10 & 11 & 12 & 13 \\
\hline$\left|T_{(2, n)}\right|$ & 2 & 5 & 8 & 15 & 27 & 52 & 100 & 197 & 389 & 774 & 1542 & 3079 & 6151 \\
$\left|\widehat{T}_{(2, n)}\right|$ & 0 & 3 & 3 & 7 & 12 & 25 & 48 & 97 & 192 & 385 & 768 & 1537 & 3072 \\
\hline
\end{tabular}

It is not difficult to see that

$$
\begin{aligned}
\widehat{B} & \cap \widehat{C}=\left\{x^{2}\left(f(x)+x^{n-2}\right) \mid f(x) \in \mathbb{F}_{2}[x]_{n-3}, f(1)\right. \\
& =1\}
\end{aligned}
$$

and, hence,

$$
|\widehat{B} \cap \widehat{C}|=2^{n-3}
$$

Therefore, by (18), we have

$$
\begin{aligned}
\left|\widehat{T}_{(2, n)}\right| & = \begin{cases}1+2^{n-2}+2^{n-2}-2^{n-3} & \text { if } n \text { is even, } \\
2^{n-2}+2^{n-2}-2^{n-3} & \text { if } n \text { is odd }\end{cases} \\
& = \begin{cases}3 \cdot 2^{n-3}+1 & \text { if } n \text { is even, } \\
3 \cdot 2^{n-3} & \text { if } n \text { is odd }\end{cases}
\end{aligned}
$$

as desired.

Table 1 presents the numbers $\left|T_{(2, n)}\right|$ and $\left|\widehat{T}_{(2, n)}\right|$ for $n=$ $1,2, \ldots, 13$. The relation $\left|T_{(2, n)}\right|=\left|T_{(2, n-1)}\right|+\left|\widehat{T}_{(2, n)}\right|$ in Remark 2 can be easily seen.

\section{Punctured Polynomials over Nonbinary Finite Fields}

In this section, we focus on punctured polynomials over nonbinary finite fields. Given a prime power $q>2$, the characterization and enumeration of good punctured polynomials of degree less than or equal to 2 over $\mathbb{F}_{q}$ are completely determined. For $n \geq 3$, we construct subsets of $T_{(q, n)}$ and $\widehat{T}_{(q, n)}$ which lead to lower bounds of the cardinalities of $T_{(q, n)}$ and $\widehat{T}_{(q, n)}$, respectively.

Theorem 8. If $q>2$ is a prime power, then $T_{(q, 1)}=\mathbb{F}_{q}$.

Proof. By the definition, $\mathbb{F}_{q} \subseteq T_{(q, 1)}$. Let $f(x)=a x+b \in T_{(q, 1)}$. Since $f_{(0)}(x)=a$ has a root in $\mathbb{F}_{q}$, we have $a=0$. Hence, $f(x)=b \in \mathbb{F}_{q}$ as desired.

The next corollary follows immediately from Theorem 8.

Corollary 9. If $q>2$ is a prime power, then the following statements hold:
(i) $\left|T_{(q, 1)}\right|=q$.
(ii) $\widehat{T}_{(q, 1)}=\emptyset$.
(iii) $\left|\widehat{T}_{(q, 1)}\right|=0$.

Theorem 10. Let $q>2$ be a prime power. Then

$$
\begin{aligned}
& T_{(q, 2)} \\
& =\left\{a_{2} x^{2}+a_{1} x+a_{0} \mid\left(a_{2}, a_{1}, a_{0}\right) \in \mathbb{F}_{q}^{*} \times \mathbb{F}_{q}^{*} \times \mathbb{F}_{q}\right\} \\
& \quad \cup\left\{a_{2} x^{2} \mid a_{2} \in \mathbb{F}_{q}^{*}\right\} \cup \mathbb{F}_{q} .
\end{aligned}
$$

Proof. Let $A=\left\{a_{2} x^{2}+a_{1} x+a_{0} \mid\left(a_{2}, a_{1}, a_{0}\right) \in \mathbb{F}_{q}^{*} \times \mathbb{F}_{q}^{*} \times \mathbb{F}_{q}\right\}$ and $B=\left\{a_{2} x^{2} \mid a_{2} \in \mathbb{F}_{q}^{*}\right\}$.

Let $f(x) \in A \cup B \cup \mathbb{F}_{q}$. We write $f(x)=a_{2} x^{2}+a_{1} x+a_{0}$ and consider the proof as two cases.

Case $1(f(x) \in A)$. We have $f_{(0)}(x)=a_{2} x+a_{1}, f_{(1)}(x)=$ $a_{2} x+a_{0}$, and $f_{(2)}(x)=a_{1} x+a_{0}$. Since $a_{2}$ and $a_{1}$ are nonzero, it follows that $-a_{1} a_{2}^{-1},-a_{0} a_{2}^{-1}$, and $-a_{0} a_{1}^{-1}$ are roots of $f_{(0)}(x)$, $f_{(1)}(x)$, and $f_{(2)}(x)$, respectively. Hence, $f(x) \in T_{(q, 2)}$.

Case $2(f(x) \in B)$. Then $f(x)=a_{2} x^{2}$ for some $a_{2} \in \mathbb{F}_{q}^{*}$. It follows that 0 is a root of $f_{(0)}(x)=a_{2} x, f_{(1)}(x)=a_{2} x$, and $f_{(2)}(x)=0$. Therefore, $f(x) \in T_{(q, 2)}$.

Case $3\left(f(x) \in \mathbb{F}_{q}\right)$. Then, by the definition, $f(x) \in T_{(q, 2)}$.

On the other hand, let $f(x)=a_{2} x^{2}+a_{1} x+a_{0} \in T_{(q, 2)}$. If $a_{2}=0$, then $f(x)=a_{1} x+a_{0} \in T_{(q, 1)}$, and, hence, $f(x)=a_{0} \in$ $\mathbb{F}_{q}$ by Theorem 8. Assume that $a_{2} \neq 0$. Then $f_{(0)}(x)=a_{2} x+a_{1}$, $f_{(1)}(x)=a_{2} x+a_{0}$, and $f_{(2)}(x)=a_{1} x+a_{0}$ have a root in $\mathbb{F}_{q}$.

Case $1\left(a_{1}=0\right)$. We have that $f_{(2)}(x)=a_{0}$ has a root in $\mathbb{F}_{q}$ which implies that $a_{0}=0$. Therefore, $f(x)=a_{2} x^{2} \in B$.

Case $2\left(a_{1} \neq 0\right)$. Since $f_{(0)}(x)=a_{2} x+a_{1}$ has a root in $\mathbb{F}_{q}$, we have $a_{2} \neq 0$. Hence, $f(x) \in A$.

From the two cases, it can be concluded that $f(x) \in A \cup$ $B \cup \mathbb{F}_{q}$.

As desired, we have $T_{(q, 2)}=A \cup B \cup \mathbb{F}_{q}$.

Corollary 11. If $q>2$ is a prime power, then

$$
\left|T_{(q, 2)}\right|=q\left(q^{2}-2 q+3\right)-1
$$

Proof. Let $A=\left\{a_{2} x^{2}+a_{1} x+a_{0} \mid\left(a_{2}, a_{1}, a_{0}\right) \in \mathbb{F}_{q}^{*} \times \mathbb{F}_{q}^{*} \times\right.$ $\left.\mathbb{F}_{q}\right\}$ and $B=\left\{a_{2} x^{2} \mid a_{2} \in \mathbb{F}_{q}^{*}\right\}$ be defined as in the proof of Theorem 10. It is not difficult to see that $A, B$, and $\mathbb{F}_{q}$ are disjoint. By Theorem 10, we have

$$
\begin{aligned}
\left|T_{(q, 2)}\right| & =\left|A \cup B \cup \mathbb{F}_{q}\right|=|A|+|B|+\left|\mathbb{F}_{q}\right| \\
& =(q-1)(q-1) q+(q-1)+q \\
& =q\left(q^{2}-2 q+3\right)-1
\end{aligned}
$$

as desired. 
Corollary 12. If $q$ is a prime power, then

$$
\begin{aligned}
\widehat{T}_{(q, 2)} & \\
= & \left\{a_{2} x^{2}+a_{1} x+a_{0} \mid\left(a_{2}, a_{1}, a_{0}\right) \in \mathbb{F}_{q}^{*} \times \mathbb{F}_{q}^{*} \times \mathbb{F}_{q}\right\} \\
& \cup\left\{a_{2} x^{2} \mid a_{2} \in \mathbb{F}_{q}^{*}\right\} .
\end{aligned}
$$

Proof. From Theorem 10, it is not difficult to see that the polynomials of degree less than 2 in $T_{(q, 2)}$ are $f(x)=a \in \mathbb{F}_{q}$. Hence, the result follows.

Corollary 13. If $q$ is a prime power, then

$$
\left|\widehat{T}_{(q, 2)}\right|=(q-1)\left(q^{2}-q+1\right) \text {. }
$$

Proof. From Corollaries 11 and 12, it follows that

$$
\begin{aligned}
\left|\widehat{T}_{(q, 2)}\right| & =\left|T_{(q, 2)}\right|-q=q\left(q^{2}-2 q+3\right)-1-q \\
& =(q-1)\left(q^{2}-q+1\right) .
\end{aligned}
$$

In the case where $n \geq 3$, determining the sets $T_{(q, n)}$ and $\widehat{T}_{(q, n)}$ is more tedious and complicated. For these cases, we give constructive lower bounds of $\left|T_{(q, n)}\right|$ and $\left|\widehat{T}_{(q, n)}\right|$.

The following results are important tools in constructing lower bounds of $\left|T_{(q, n)}\right|$ and $\left|\widehat{T}_{(q, n)}\right|$.

Theorem 14 (see [14, Page 588]). Let $n$ be a positive integer and let $q$ be a prime power. Then the number of monic irreducible polynomials of degree $n$ in $\mathbb{F}_{q}[x]$ is

$$
L(n, q)=\frac{1}{n} \sum_{d \mid n} \mu(d) q^{n / d},
$$

where

$$
\begin{aligned}
& \mu(n) \\
& = \begin{cases}1 & \text { if } n=1, \\
0 & \text { if } n \text { contains a repeated prime factor, } \\
(-1)^{r} & \text { if } n \text { is a product of } r \text { distinct primes }\end{cases}
\end{aligned}
$$

is the Möbius function.

Theorem 15 (see [3, Section 4.2, Theorem 1]). Let $f(x)$ be a polynomial of degree 2 or 3 in $\mathbb{F}_{q}[x]$. Then $f(x)$ is reducible if and only if $f(x)$ has a root in $\mathbb{F}_{q}[x]$.

Theorem 16. If $q>2$ is a prime power, then

$$
\left|\widehat{T}_{(q, 3)}\right| \geq \frac{1}{2}(q-1) q(q+1) .
$$

Proof. Let $S:=\left\{x f(x) \mid f(x) \in \widehat{\mathbb{F}}_{q}[x]_{2}\right.$ and $f(x)$ has a root in $\mathbb{F}_{q}$.
First, we show that $S \subseteq \widehat{T}_{(q, 3)}$. Let $g(x)=x\left(a_{2} x^{2}+a_{1} x+\right.$ $\left.a_{0}\right) \in S$, where $a_{2} \in \mathbb{F}_{q}^{*}$ and $a_{1}, a_{0} \in \mathbb{F}_{q}$. Then $g_{(0)}(x)=a_{2} x^{2}+$ $a_{1} x+a_{0}$ has a root in $\mathbb{F}_{q}$ and 0 is a root of

$$
\begin{aligned}
& g_{(1)}(x)=a_{2} x^{2}+a_{1} x, \\
& g_{(2)}(x)=a_{2} x^{2}+a_{0} x, \\
& g_{(3)}(x)=a_{1} x^{2}+a_{0} x .
\end{aligned}
$$

Hence, $g(x) \in \widehat{T}_{(q, 3)}$ is good punctured.

By Theorem 14, the number of monic irreducible polynomials of degree 2 over $\mathbb{F}_{q}$ is

$$
\begin{aligned}
L(q, 2) & =\frac{1}{2} \sum_{d \mid 2} \mu(d) q^{2 / d}=\frac{1}{2}\left(\mu(1) q^{2}+\mu(2) q^{1}\right) \\
& =\frac{1}{2}\left(q^{2}-q\right) .
\end{aligned}
$$

Hence, the number of irreducible polynomials of degree 2 over $\mathbb{F}_{q}$ is

$$
\frac{1}{2}(q-1)\left(q^{2}-q\right)
$$

By Theorem 15, the number of polynomials of degree 2 over $\mathbb{F}_{q}$ having a root in $\mathbb{F}_{q}$ is

$$
\begin{aligned}
|S| & =(q-1) q^{2}-\frac{1}{2}(q-1)\left(q^{2}-q\right) \\
& =\frac{1}{2}(q-1) q(q+1) .
\end{aligned}
$$

Since $S \subseteq \widehat{T}_{(q, 3)}$, we have

$$
\left|\widehat{T}_{(q, 3)}\right| \geq|S|=\frac{1}{2}(q-1) q(q+1)
$$

as desired.

Corollary 17. If $q>2$ is a prime power, then

$$
\left|T_{(q, 3)}\right| \geq \frac{3 q^{3}-4 q^{2}+5 q-2}{2} .
$$

Proof. By Corollary 11 and Theorem 16, we have

$$
\begin{aligned}
& \left|T_{(q, 2)}\right|=q\left(q^{2}-2 q+3\right)-1, \\
& \left|\widehat{T}_{(q, 3)}\right| \geq \frac{1}{2}(q-1) q(q+1) .
\end{aligned}
$$

Hence, by Remark 2, we have the relation

$$
\begin{aligned}
\left|T_{(q, 3)}\right| & =\left|T_{(q, 2)}\right|+\left|\widehat{T}_{(q, 3)}\right| \\
& \geq q\left(q^{2}-2 q+3\right)-1+\frac{1}{2}(q-1) q(q+1) \\
& =\frac{3 q^{3}-4 q^{2}+5 q-2}{2} .
\end{aligned}
$$


Theorem 18. If $q>2$ is a prime power, then

$$
\left|\widehat{T}_{(q, 4)}\right| \geq \frac{1}{2}(q-1) q\left(q^{2}+1\right) .
$$

Proof. Let $S:=\left\{x f(x) \mid f(x) \in \widehat{\mathbb{F}}_{q}[x]_{3}\right.$ and $f(x)$ has a root in $\left.\mathbb{F}_{q}\right\}$.

First, we show that $S \subseteq \widehat{T}_{(q, 4)}$. Let $g(x)=x\left(a_{3} x^{3}+a_{2} x^{2}+\right.$ $\left.a_{1} x+a_{0}\right) \in S$, where $a_{3} \in \mathbb{F}_{q}^{*}$ and $a_{2}, a_{1}, a_{0} \in \mathbb{F}_{q}$. Then $g_{(0)}(x)=$ $a_{3} x^{3}+a_{2} x^{2}+a_{1} x+a_{0}$ has a root in $\mathbb{F}_{q}$ and 0 is a root of

$$
\begin{aligned}
& g_{(1)}(x)=a_{3} x^{3}+a_{2} x^{2}+a_{1} x, \\
& g_{(2)}(x)=a_{3} x^{3}+a_{2} x^{2}+a_{0} x, \\
& g_{(3)}(x)=a_{3} x^{3}+a_{1} x^{2}+a_{0} x, \\
& g_{(4)}(x)=a_{2} x^{3}+a_{1} x^{2}+a_{0} x .
\end{aligned}
$$

Therefore, $g(x) \in \widehat{T}_{(q, 4)}$ is good punctured as desired.

By Theorem 14, the number of monic irreducible polynomials of degree 3 over $\mathbb{F}_{q}$ is

$$
\begin{aligned}
L(q, 3) & =\frac{1}{3} \sum_{d \mid 3} \mu(d) q^{3 / d}=\frac{1}{2}\left(\mu(1) q^{3}+\mu(3) q^{1}\right) \\
& =\frac{1}{2}\left(q^{3}-q\right) .
\end{aligned}
$$

Hence, the number of irreducible polynomials of degree 3 over $\mathbb{F}_{q}$ is

$$
\frac{1}{2}(q-1)\left(q^{3}-q\right)
$$

By Theorem 15, the number of polynomials of degree 3 over $\mathbb{F}_{q}$ having a root in $\mathbb{F}_{q}$ is

$$
\begin{aligned}
|S| & =(q-1) q^{3}-\frac{1}{2}(q-1)\left(q^{3}-q\right) \\
& =\frac{1}{2}(q-1) q\left(q^{2}+1\right) .
\end{aligned}
$$

Since $S \subseteq \widehat{T}_{(q, 4)}$, we have

$$
\left|\widehat{T}_{(q, 4)}\right| \geq|S|=\frac{1}{2}(q-1) q\left(q^{2}+1\right)
$$

as desired.

Corollary 19. If $q>2$ is a prime power, then

$$
\left|T_{(q, 4)}\right| \geq \frac{q^{4}+2 q^{3}-3 q^{2}+4 q-2}{2} .
$$

Proof. By Corollary 17, we have

$$
\left|T_{(q, 3)}\right| \geq \frac{3 q^{3}-4 q^{2}+5 q-2}{2} .
$$

From Theorem 18, we have

$$
\left|\widehat{T}_{(q, 4)}\right| \geq \frac{1}{2}(q-1) q\left(q^{2}+1\right) .
$$

Hence, by Remark 2, we have the relation

$$
\begin{aligned}
\left|T_{(q, 4)}\right| & =\left|T_{(q, 3)}\right|+\left|\widehat{T}_{(q, 4)}\right| \\
& \geq \frac{3 q^{3}-4 q^{2}+5 q-2}{2}+\frac{1}{2}(q-1) q\left(q^{2}+1\right) \\
& =\frac{q^{4}+2 q^{3}-3 q^{2}+4 q-2}{2} .
\end{aligned}
$$

Theorem 20. Let $q>2$ be a prime power and let $n \geq 5$ be an integer. Then

$$
\left|T_{(q, n)}\right| \geq q^{n-1}+q^{3}-2 q^{2}+2 q .
$$

Proof. Let $A=\left\{x^{2} f(x) \mid f(x) \in \mathbb{F}_{q}[x]_{n-2}\right\}$.

First, we show that $A \cup T_{(q, 2)} \subseteq T_{(q, n)}$. Clearly, $T_{(q, 2)} \subseteq$ $T_{(q, n)}$. Let $g(x) \in A$. Then 0 is a root of $g_{(i)}(x)$ for all $i=$ $0,1, \ldots, \operatorname{deg}(g(x))$, and, hence, $g(x) \in T_{(q, n)}$. Note that $A \cap$ $T_{(q, 2)}=\left\{a x^{2} \mid a \in \mathbb{F}_{q}^{*}\right\}$. By Corollary 11, we have

$$
\left|T_{(q, 2)}\right|=q\left(q^{2}-2 q+2\right)
$$

Therefore, consider

$$
\begin{aligned}
\left|T_{(q, n)}\right| & \geq|A|+\left|T_{(q, 2)}\right|-\left|A \cap T_{(q, 2)}\right| \\
& =q^{n-1}+q\left(q^{2}-2 q+3\right)-1-(q-1) \\
& =q^{n-1}+q^{3}-2 q^{2}+2 q .
\end{aligned}
$$

Corollary 21. Let $q>2$ be a prime power and let $n \geq 5$ be an integer. Then $\left|\widehat{T}_{(q, n)}\right| \geq(q-1) q^{n-2}$.

Proof. The set of elements in $A$ of degree $n$ in the proof of Theorem 20 is $\widehat{A}=\left\{x^{2} f(x) \mid f(x) \in \widehat{\mathbb{F}}_{q}[x]_{n-2}\right\}$. By Theorem 20, we have

$$
\left|\widehat{T}_{(q, n)}\right| \geq|\widehat{A}| \geq(q-1) q^{n-2} .
$$

\section{Conclusion and Open Problems}

The concepts of punctured polynomials and good punctured polynomials are introduced. Over the finite field $\mathbb{F}_{2}$, the complete characterization and enumeration of such polynomials are given. Over nonbinary finite fields, the good punctured polynomials of degree less than or equal to 2 are completely determined. For $n \geq 3$, constructive lower bounds of the number of good punctured polynomials of degree $n$ are given. 
In general, the following related problems are also interesting:

(1) Determine the sets $\widehat{T}_{(q, n)}$ and $\widehat{T}_{(q, n)}$, where $q>2$ is a prime power and $n \geq 3$ is an integer.

(2) Determine the exact values of $\left|\widehat{T}_{(q, n)}\right|$ and $\left|\widehat{T}_{(q, n)}\right|$, where $q>2$ is a prime power and $n \geq 3$ is an integer.

(3) Improve lower bounds of $\left|\widehat{T}_{(q, n)}\right|$ and $\left|\widehat{T}_{(q, n)}\right|$, where $q>2$ is a prime power and $n \geq 3$ is an integer.

(4) Characterize and enumerate the good punctured polynomials of degree $n$ over the real numbers $\mathbb{R}$ or over the rational numbers $\mathbb{Q}$.

\section{Competing Interests}

The authors declare that there are no competing interests regarding the publication of this paper.

\section{Acknowledgments}

This research is supported by the Thailand Research Fund under Research Grant TRG5780065.

\section{References}

[1] B. Kalantari, Polynomial Root-Finding and Polynomiography, World Scientific Publishing, River Edge, NJ, USA, 2008.

[2] V. Y. Pan and A.-L. Zheng, "New progress in real and complex polynomial root-finding," Computers \& Mathematics with Applications, vol. 61, no. 5, pp. 1305-1334, 2011.

[3] W. K. Nicholson, Introduction to Abstract Algebra, John Wiley \& Sons, New York, NY, USA, 2012.

[4] C. C. Pinter, A Book of Abstract Algebra, Dover, Mineola, NY, USA, 2nd edition, 2010.

[5] R. Lidl and H. Niederreiter, Finite Fields, Cambridge University Press, Cambridge, UK, 1997.

[6] E. Sangwisut, S. Jitman, S. Ling, and P. Udomkavanich, "Hulls of cyclic and negacyclic codes over finite fields," Finite Fields and their Applications, vol. 33, pp. 232-257, 2015.

[7] A. Akbary and Q. Wang, "On some permutation polynomials over finite fields," International Journal of Mathematics and Mathematical Sciences, vol. 2005, no. 16, pp. 2631-2640, 2005.

[8] R. A. Mollin and C. Small, "On permutation polynomials over finite fields," International Journal of Mathematics and Mathematical Sciences, vol. 10, no. 3, pp. 535-543, 1987.

[9] A. Sahni and P. T. Sehgal, "Hermitian self-orthogonal constacyclic codes over finite fields," Journal of Discrete Mathematics, vol. 2014, Article ID 985387, 7 pages, 2014.

[10] E. R. Berlekamp, "Factoring polynomials over finite fields," The Bell System Technical Journal, vol. 46, pp. 1853-1859, 1967.

[11] M. C. R. Butler, "On the reducibility of polynomials over a finite field," The Quarterly Journal of Mathematics, vol. 5, pp. 102-107, 1954.

[12] J.-M. Couveignes and R. Lercier, "Fast construction of irreducible polynomials over finite fields," Israel Journal of Mathematics, vol. 194, no. 1, pp. 77-105, 2013.
[13] A. Knopfmacher and J. Knopfmacher, "Counting polynomials with a given number of zeros in a finite field," Linear and Multilinear Algebra, vol. 26, no. 4, pp. 287-292, 1990.

[14] D. S. Dummit and R. M. Foote, Abstract Algebra, John Wiley \& Sons, New York, NY, USA, 3rd edition, 2003. 


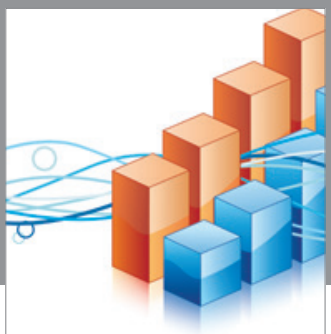

Advances in

Operations Research

vatem alat4

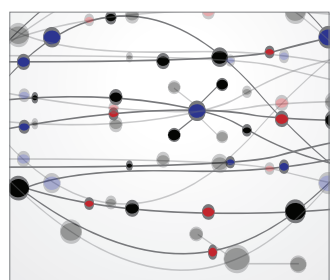

\section{The Scientific} World Journal
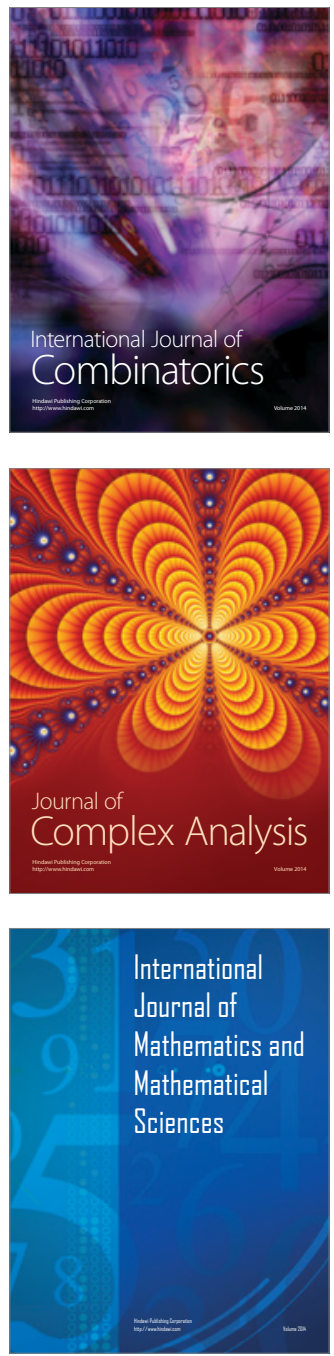
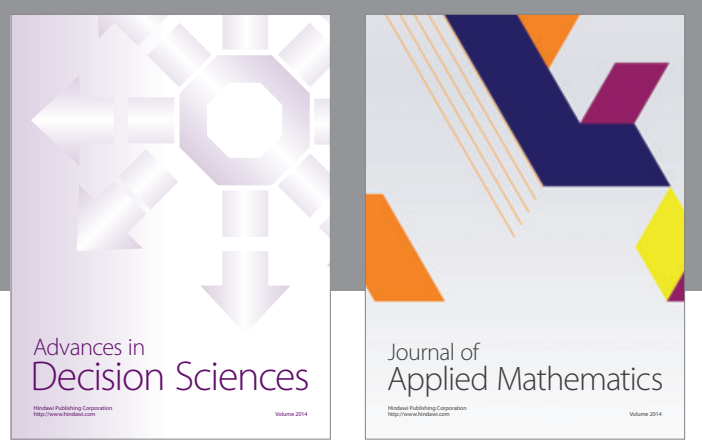

Algebra

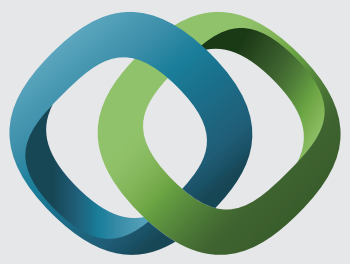

\section{Hindawi}

Submit your manuscripts at

http://www.hindawi.com
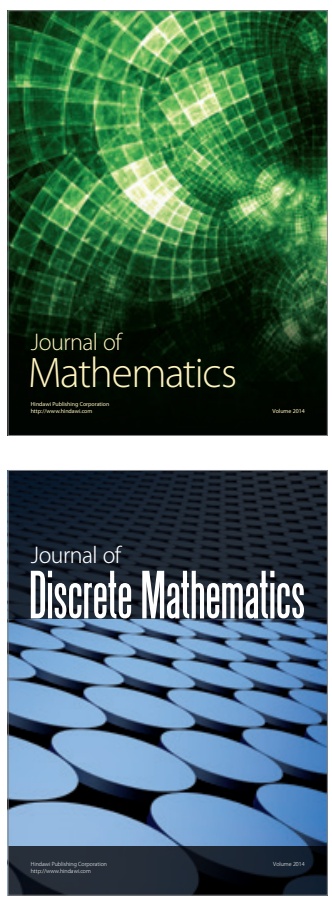

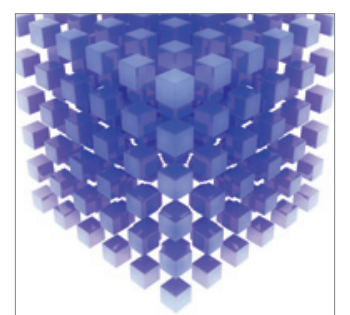

Mathematical Problems in Engineering
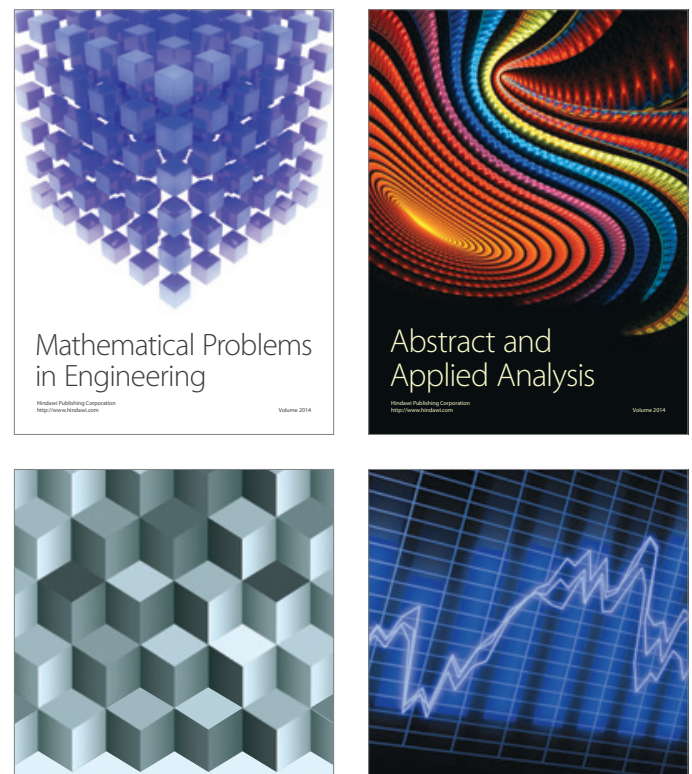

Journal of

Function Spaces

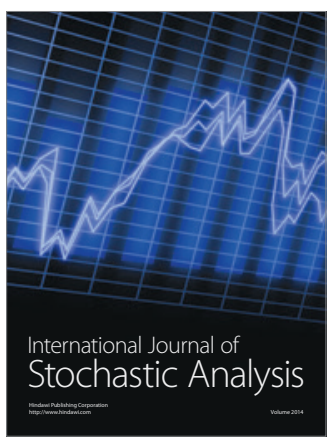

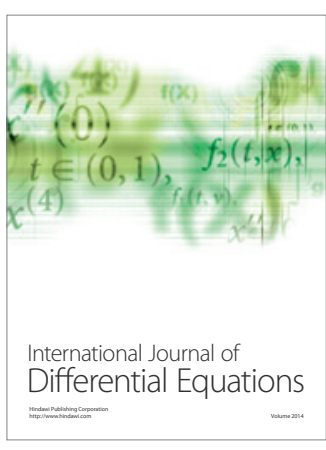
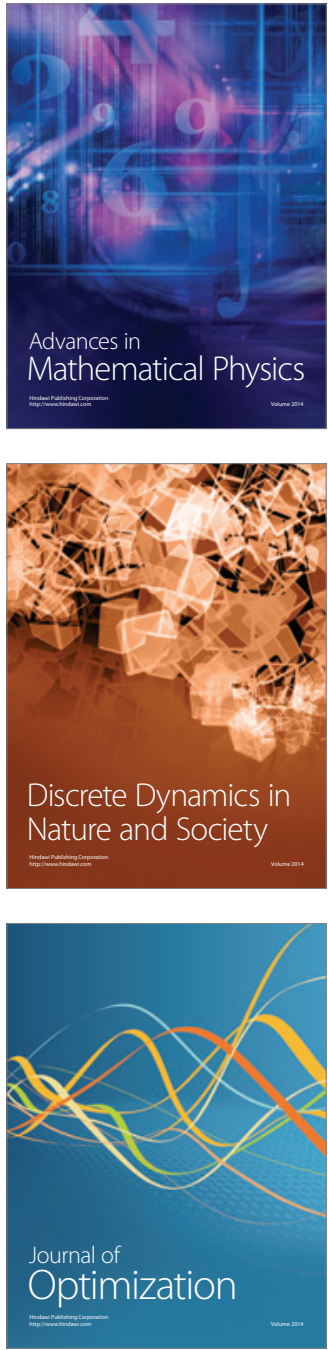